\title{
Imatinib in the treatment of chronic myeloid leukemia in children and adolescents is effective and well tolerated: Report of the Polish Pediatric Study Group for the Treatment of Leukemias and Lymphomas
}

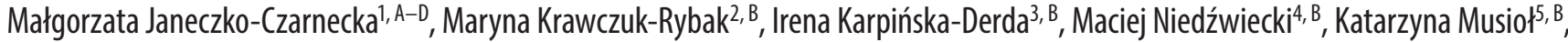 \\ Magdalena Ćwiklińska, ${ }^{6, B}$, Katarzyna Drabko7, B, Katarzyna Myck8 ${ }^{8, B}$, Tomasz Ociepa ${ }^{9,8}$, Katarzyna Pawelec ${ }^{10, B}$, \\ Danuta Januszkiewicz-Lewandowska ${ }^{11, B}$, Marek Ussowicz ${ }^{1, B, E, F}$, Blanka Rybka ${ }^{1, B}$, Renata Ryczan-Krawczyk ${ }^{11, B}$, \\ Andrzej Kołtan ${ }^{12, B}$, Grażyna Karolczyk ${ }^{13, B}$, Agnieszka Zaucha-Prażmo ${ }^{7, B}$, Wanda Badowska ${ }^{14, B}$, Krzysztof Kałwak ${ }^{11, A, E, F}$ \\ ${ }^{1}$ Department and Clinic of Pediatric Bone Marrow Transplantation, Oncology and Hematology, Wroclaw Medical University, Poland \\ 2 Department of Pediatric Oncology and Hematology, Medical University of Bialystok, Poland \\ ${ }^{3}$ Department of Pediatrics, Hematology and Oncology, Dr Edward Hanke Chorzów Center of Pediatrics and Oncology, Poland \\ ${ }^{4}$ Department and Clinic of Pediatrics, Hematology and Oncology, Medical University of Gdańsk, Poland \\ ${ }^{5}$ Silesian Children's Health Center, Department of Oncology, Hematology and Pediatric Chemotherapy, Medical University of Silesia in Katowice, Poland \\ ${ }^{6}$ Department of Oncology and Hematology, Polish-American Institute of Pediatrics, Jagiellonian University Medical College, Kraków, Poland \\ ${ }^{7}$ Department of Pediatric Hematology, Oncology and Transplantology, Medical University of Lublin, Poland \\ ${ }^{8}$ Department of Pediatrics, Oncology, Hematology and Diabetology, Medical University of Lodz, Poland \\ ${ }^{9}$ Department of Pediatrics, Pediatric Hematology and Oncology, Pomeranian Medical University in Szczecin, Poland \\ ${ }^{10}$ Department and Clinic of Pediatrics, Hematology and Oncology, Medical University of Warsaw, Poland \\ "Department of Oncology, Hematology and Pediatric Transplantatology, Poznan University of Medical Sciences, Poland \\ ${ }^{12}$ Department of Pediatrics, Hematology and Oncology, Nicolaus Copernicus University in Toruń, Collegium Medicum in Bydgoszcz, Poland \\ ${ }^{13}$ Department of Hematooncology, Wladyslaw Buszkowski Provincial Specialist Children's Hospital in Kielce, Poland \\ ${ }^{14}$ Department and Clinic of Pediatric Hematology and Oncology, Prof. Stanisław Popowski Provincial Specialist Childrens' Hospital in Olsztyn, Poland \\ A - research concept and design; $\mathrm{B}$ - collection and/or assembly of data; $\mathrm{C}$ - data analysis and interpretation \\ $D$ - writing the article; $E$ - critical revision of the article; $F$ - final approval of the article
}

Address for correspondence

Małgorzata Janeczko-Czarnecka

E-mail:ml.janeczko@gmail.com

Funding sources

None declared

Conflict of interest

None declared

\section{Acknowledgements}

The authors thank Jennifer Grbevski and Nicole

Grbevski for their careful English language editing

Received on May 28, 2016

Reviewed on June 7, 2016

Accepted on 0ctober 27, 2016

DOI

10.17219/acem/66462

Copyright

Copyright by Author(s)

This is an article distributed under the terms of the Creative Commons Attribution Non-Commercial License (http://creativecommons.org/licenses/by-nc-nd/4.0/)

\section{Abstract}

Background. Chronic myeloid leukemia (CML) constitutes only 2-3\% of all leukemias in pediatric patients. Philapelphia chromosome and BCR-ABL fusion are genetic hallmarks of CML, and their presence is crucial for targeted molecular therapy with tyrosine kinase inhibitors (TKIs), which replaced hematopoietic stem cell transplantation (HSCT) as a standard first-line therapy. The disease in pediatric population is rare, and despite molecular and clinical similarities to CML in adults, different approach is needed, due to the long lifetime expectancy and distinct developmental characteristics of affected children.

Objectives. The objective of this study is to evaluate treatment with imatinib in Polish pediatric patients with CML.

Material and methods. We analyzed the results of treatment with imatinib in 57 pediatric patients (June 2006 - January 2016) from 14 Polish pediatric hematology and oncology centers.

Results. In the study group, 40 patients continued imatinib (median follow-up: 23.4 months), while in 17 the treatment was terminated (median follow-up: 15.1 months) due to therapy failure. In the latter group, 13 patients underwent HSCT, while 4 switched to second-generation TKIs. The 5 -year overall survival rate (OS) in the study group was $96 \%$, and the 5 -year event-free survival (EFS) was $81 \%$.

Conclusions. Our results confirm that the introduction of TKI therapy has revolutionized the treatment of CML in the pediatric population by replacing the previous method of treatment with HSCT and allowing a high percentage of $O S$ and $E F S$.

Key words: children, imatinib, adolescents, chronic myeloid leukemia 


\section{Introduction}

Chronic myeloid leukemia (CML) is a myeloproliferative neoplasm typically diagnosed in the adult population and is relatively rare in children with the incidence of 0.6-1.2 million per year. ${ }^{1} \mathrm{CML}$ is a clonal disorder of hematopoietic progenitor cells resulting from the balanced translocation $(9 ; 22)$ called the Philadelphia chromosome $(\mathrm{Ph})$ at the molecular level, resulting in the formation of a fusion gene $B C R-A B L$. The BCR-ABL protein encoded by the fusion gene exhibits tyrosine kinase activity and promotes uncontrolled proliferation of pluripotent stem cells in bone marrow (BM). CML has a 3-phase course: chronic phase (CML-CP); accelerated phase (CML-AP); and blast crisis phase (CML-BC). CML is most commonly diagnosed in the CML-CP and only in about $10 \%$ of cases in advanced phases: CML-AP or CML-BC. ${ }^{2}$

Before the implementation of imatinib, hydroxyurea + /interferon alpha remained the first-line treatment of CML, followed by HSCT after achieving hematologic remission. ${ }^{3}$ The identification of tyrosine kinase inhibitors (TKI) with BCR-ABL blocking ability revolutionized the CML therapy due to pharmacological control of leukemic clone. TKI-based therapy has proved to be very effective and quickly led to the withdrawal of HSCT as the first-line treatment. A first-generation TKI - imatinib was approved for the treatment of adult patients in 2001. Then, in 2003, it was approved, for the therapy in children. It should be emphasized that treatment with TKI is not a way to cure CML, as in most patients leukemia cells are still present. However, a unique feature of these drugs is a significant reduction of the risk of CML progression. ${ }^{2}$ In the era of TKIs, the CML-CP can last beyond 20 years.

The recommended starting dose of imatinib for children is $260-300 \mathrm{mg} / \mathrm{m}^{2}$ (max daily dose: $400 \mathrm{mg}$ ) in CP, $400 \mathrm{mg} / \mathrm{m}^{2}$ (max daily dose: $600 \mathrm{mg}$ ) in AP, and $500 \mathrm{mg} / \mathrm{m}^{2}$ (max daily dose: $800 \mathrm{mg}$ ) in the blastic phase. ${ }^{3}$ In recent years, due to the development of the targeted therapy and the implementation of TKIs in pediatric patients, there has been significant progress in the treatment of CML, but the data is relatively limited due to low incidence of CML. One should remember that long-term side effects of TKI therapy may occur, because the drug has only been in use for approx. 15 years. Despite the excellent results of imatinib therapy, one should not forget about HSCT. It is the only method for obtaining definite cure of CML. HSCT is the first-line treatment in patients with CML who have become resistant to the first-and second-generation TKIs, or when serious side effects of the therapy occur and there is a matched donor available. In some cases, the preference of HSCT by the patient or his/her parents is also very important. It is worth noting that HSCT should be considered more frequently in patients diagnosed with CML before puberty due to growth impairment after TKI therapy. The transplant is in fact the only method that can lead to the cure of this disease.

\section{Material and methods}

We conducted a retrospective, nation-wide analysis of the imatinib therapy results in children and adolescents with CML in Poland. The study group consisted of $57 \mathrm{pa-}$ tients $(\mathrm{M}=35, \mathrm{~F}=22)$ from 14 Polish pediatric hematology and oncology centers treated in 2006-2016. The majority of patients $(n=54)$ were diagnosed in the chronic phase and only 3 patients in the accelerated phase. The diagnosis of CML was made according to the $4^{\text {th }}$ edition of WHO classification of hematopoietic and lymphoid tissues, with mandatory molecular confirmation of BCR-ABL fusion. The first-line treatment with imatinib was performed accordingly to previous I-BFM recommendations. Patients and/or their legal guardians signed the appropriate consents.

The effectiveness of TKI therapy was evaluated on the basis of "milestones" of the therapy: complete hematologic remission (CHR); complete cytogenetic response (CCyR); and major molecular response (MMR). CHR was defined as leukocyte count $<10 \times 10^{3} / \mu \mathrm{L},<5 \%$ basophils and $<450 \times$ $10^{3} / \mu \mathrm{L}$ platelets in peripheral blood, absence of myelocytes, promyelocytes and blasts in the peripheral blood, and nonpalpable spleen during physical examination. Starting from the moment of CML diagnosis blood counts with blood smear should be performed every 15 days until achieving $\mathrm{CHR}$, and then at least once every 3 months. Complete cytogenetic response (CCyR) was defined as the absence of $\mathrm{Ph}(+)$ cells in the bone marrow in classical cytogenetics or the FISH method, and evaluated within 12 months of the treatment.

Partial cytogenetic response (PCyR) was the presence of $1-35 \% \mathrm{Ph}(+)$ cells in the bone marrow. Minor and minimal cytogenetic response was the presence of $36-65 \%$ or $66-95 \% \mathrm{Ph}(+)$ cells in the bone marrow, respectively. Cytogenetic evaluation was performed after 3 and 6 months after the implementation of imatinib, then every 6 months until CCyR achievement, and every 12 months thereafter or in the case of treatment failure.

Molecular response was evaluated after 18 months of the treatment. Major molecular response (MMR) was defined as the BCR-ABL transcript level below $0.1 \%$ Molecular tests were performed every 3 months until MMR confirmation, and then not less than every 6 months. If there was an unsatisfactory response, testing for BCRABL kinase domain mutations was performed.

\section{Statistical analysis}

The statistical analysis was performed using STATISTICA v. 10.0. As events in the analysis of event-free survival (EFS) we considered the following: death of the patient; switch to second-generation tyrosine kinase inhibitors (2G TKIs) due to therapy failure or imatinib intolerance; proceeding with HSCT due to intolerance of imatinib and/or loss of cytogenetic or molecular response during imatinib therapy. 


\section{Results}

\section{Study group}

The median age at CML diagnosis was 13.6 years (the youngest child was 1.2 year, the oldest 17.9 years). The most commonly reported symptoms at the diagnosis were: asthenia $(\mathrm{n}=22)$; weight loss $(\mathrm{n}=18)$; abdominal pain $(\mathrm{n}=17)$; fever $(\mathrm{n}=16)$; limb pain $(\mathrm{n}=10)$; and hemorrhage $(\mathrm{n}=7)$. Other reported symptoms included ecchymoses $(\mathrm{n}=4)$; headache $(\mathrm{n}=4)$; diplopia $(\mathrm{n}=2)$; pallor $(\mathrm{n}=2)$; cough $(\mathrm{n}=2)$; priapism $(\mathrm{n}=2)$; breast pain $(\mathrm{n}=1)$; night sweats $(n=1)$; arthritis $(n=1)$; dyspnoea $(n=1)$; polydipsia $(n=1)$; hair loss $(n=1)$; and intramuscular hematoma $(n=1)$. The most frequent signs were splenomegaly $(n=43$; median size of the spleen was $5.5 \mathrm{~cm}$ below the costal margin), and hepatomegaly ( $\mathrm{n}=34$; median size of the liver was $3 \mathrm{~cm}$ below the costal margin). In 8 cases, the diagnosis was set on the basis of routine blood tests without other accompanying symptoms. Laboratory tests revealed significant hyperleukocytosis in the majority of patients (median $226.2810^{3} / \mu \mathrm{L}$, range $7.17-81010^{3} / \mu \mathrm{L}$ ) and thrombocytosis (median $47110^{3} / \mu \mathrm{L}$, range $27.9-3444.710^{3} / \mu \mathrm{L}$ ). During the follow-up period, 2 patients died, both after transplantation. One of them died on day +307 after HSCT from central nervous system aspergillosis and multiorgan failure, while the other died on day +76 after HSCT from grade IV acute Graft vs Host Disease (aGvHD) and pulmonary hemorrhage. The 5-year OS in the study group was 96\% (Fig. 1) and the 5-year EFS was 79\% (Fig. 2).

\section{Treatment with imatinib}

All pediatric patients were qualified for first-line treatment with imatinib, according to CML-PAED II and IBFM-CML protocol. Hydroxyurea was administered as pre-imatinib cytoreductive phase in 21 patients (median duration: 16 days). In 1 case, anagrelide was administered alternatively for the treatment of CML-associated thrombocytosis. The median time of imatinib implementation from the moment of diagnosis was 7 days (range 0-202 days). The median initial dose was $300 \mathrm{mg} / \mathrm{m}^{2}$ (range $220-468 \mathrm{mg} / \mathrm{m}^{2}$ ). The maximum dose was implemented in a single patient in CP, who had become resistant to the standard doses and experienced lack of CCyR and MMR in 12 and 18 months after the initiation of therapy, respectively. The dose of imatinib during therapy was modified in 23 cases. In 13 patients, the dose

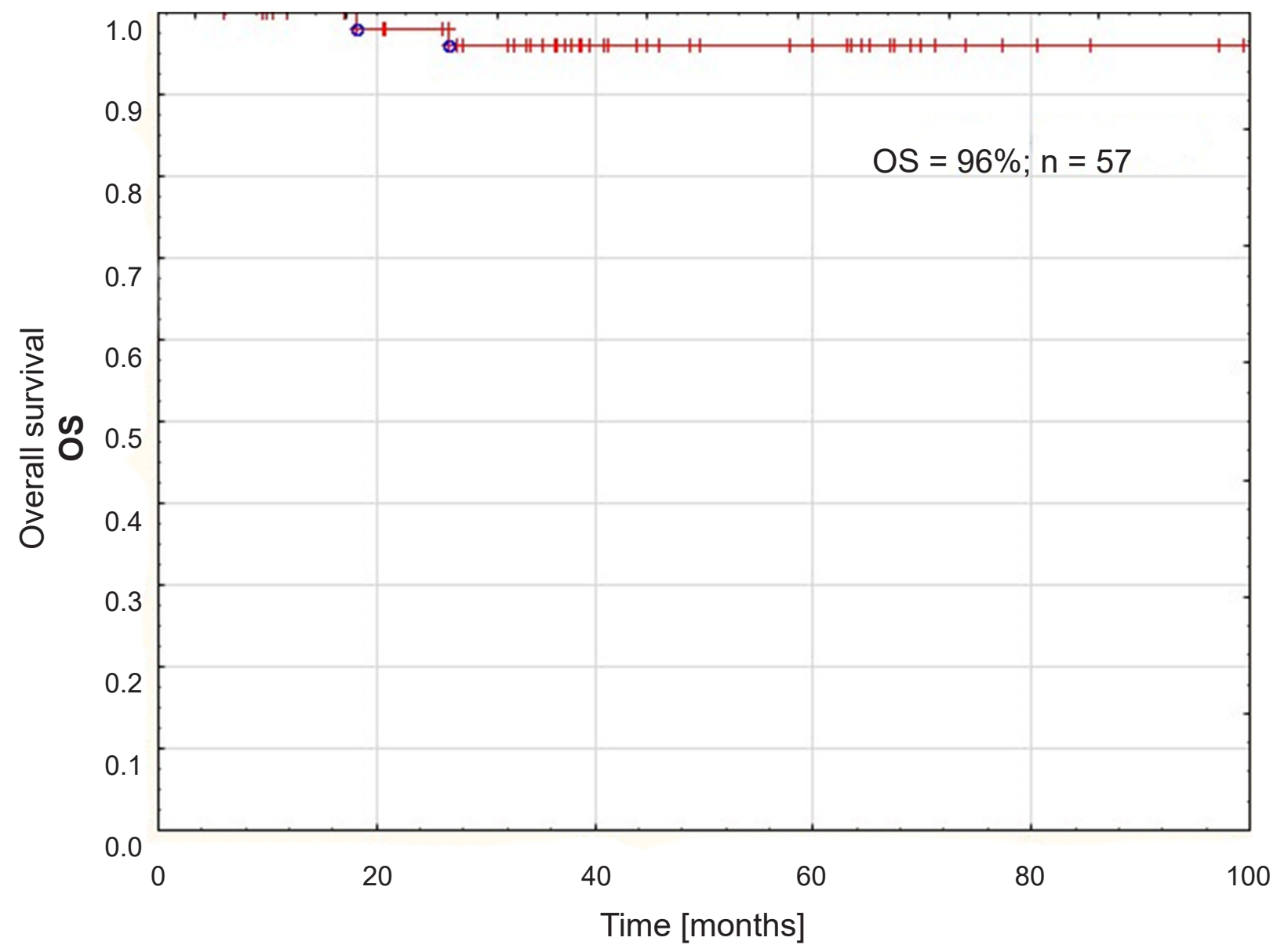

Fig. 1. 5-year overall survival (OS) in the study group ( $n=57$; alive $=55$, died $=2$ ) 
had to be increased from $300 \mathrm{mg} / \mathrm{m}^{2}$ to $400 \mathrm{mg} / \mathrm{m}^{2}$ due to resistance to imatinib therapy. In 10 patients, the imatinib dose was reduced due to toxicity $(n=8)$ or satisfactory response to a higher dose $(\mathrm{n}=2)$ (median dose after reduction: $170 \mathrm{mg} / \mathrm{m}^{2}$ ). Toxicity included myelotoxicity (WHO grade $4, \mathrm{n}=1)$; thrombocytopenia $(\mathrm{n}=1)$; leukopenia $(\mathrm{n}=5)$; and headache $(\mathrm{n}=1)$. In 4 patients, 2G TKIs were implemented after imatinib: dasatinib in 3 cases, and nilotinib in 1 case. In 19 patients, due to unsatisfactory response to imatinib, testing for BCR-ABL kinase domain mutations was performed. In 3 cases, the T315I mutation was confirmed, and all these patients underwent HSCT afterwards. In 1 case, ponatinib achieved molecular remission.

\section{Complete hematologic remission}

Within 3 months complete chematologic remission (CHR) was achieved in 57 patients. In 2 patients, we observed hematologic relapse. One patient underwent HSCT afterwards, and the other was switched to 2 G TKI-nilotinib and achieved another CHR.

\section{Complete cytogenetic response}

Within 12 months, complete cytogenetic response (CCyR) was evaluated in 45 out of 57 patients. In 4 cases, the follow-up period did not exceed 1 year, 4 patients underwent HSCT in less than 12 months since the start of the treatment, 2 patients were transferred to an adult ward before the evaluation, while in 2 patients cytogenetic response was not assessed. Within 12 months from the onset of therapy, CCyR was achieved in 31/45 patients (i.e., 69\%). After 12 months, CCyR was observed in $9 / 45$ cases (i.e., 20\%). CCyR was not achieved in 5 cases (i.e., 11\%). Out of the 5 patients who failed to achieve CCyR, 3 patients were switched to dasatinib, and 2 patients underwent HSCT afterwards.

\section{Major molecular response}

After 18 months, major molecular response (MMR) was evaluated in 46 out of 57 patients. In 5 cases, the follow-up period did not reach 1.5 year. Four patients underwent HSCT in less than 18 months since the start of the treatment,

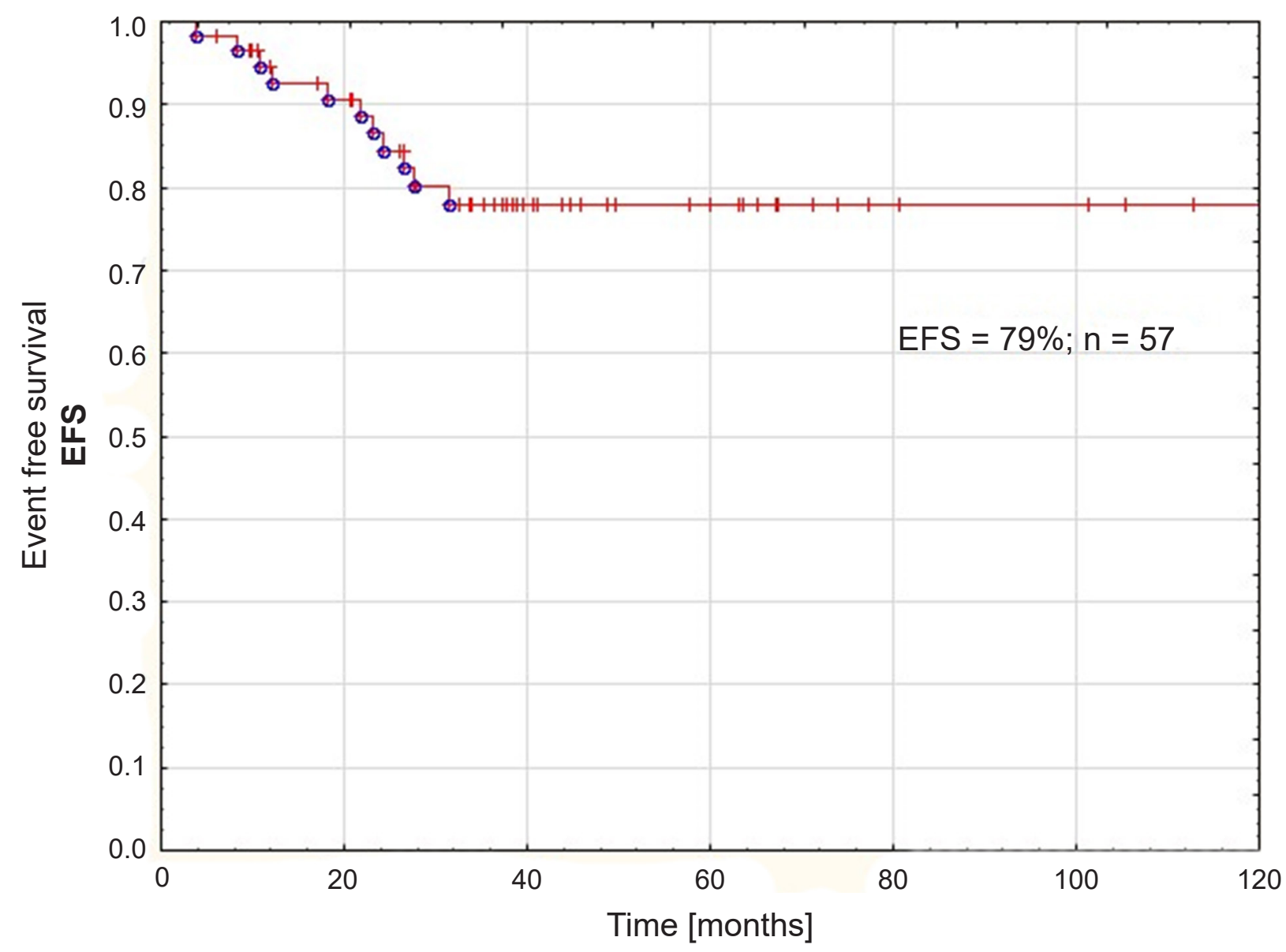

Fig. 2. 5-year event free survival (EFS) in the study group $(n=57)$

event free $=45$; events $=12$ (death of the patient; switch to 2 G TKIs due to therapy failure or imatinib intolerance; HSCT due to intolerance of imatinib and/or loss of cytogenetic or molecular response during imatinib therapy). 
and 2 patients were transferred to an adult ward. MMR was achieved in $28 / 46$ patients (i.e., $61 \%$ ) after 18 months since the beginning of the treatment. MMR later than 18 months from therapy start was seen in $8 / 46$ patients (i.e., $17 \%$ ) with median time of 24 months. MMR was not achieved in 10/46 cases (i.e., 22\%). Five of these patients underwent HSCT afterwards, and 2 were switched to $2 \mathrm{G}$ TKI-dasatinib. In 3 cases, the dose of imatinib was increased, with continuous decrease in the BCR-ABL level. Characteristics of the study group and the results of treatment are summarized in Table 1.

Table 1. Characteristics of the study group. Results of treatment with imatinib in 57 pediatric patients (June 2006 - January 2016) from 14 Polish pediatric hematology and oncology centres

\begin{tabular}{|l|c|}
\multicolumn{1}{|c|}{ Study group } & Number of patients \\
\hline Patients total & 57 \\
male & 35 \\
female & 22 \\
\hline Phase at the diagnosis & \\
chronic phase & 54 \\
accelerated phase & 3 \\
\hline Hematologic remission & \\
complete hematologic remission (CHR) & 55 \\
hematologic relapse & 2 \\
\hline Cytogenetic response evaluable & $45 / 57$ \\
CCyR within 12 months & $31(69 \%)$ \\
CCyR after 12 months & $9(20 \%)$ \\
failed to achieve CCyR & $5(11 \%)$ \\
switch to dasatinib & 2 \\
HSCT & 3 \\
\hline Molecular response evaluable & $46 / 57$ \\
MMR within 18 months & $28(61 \%)$ \\
MMR after 18 months & $8(17 \%)$ \\
failed to achieve & $10(22 \%)$ \\
HSCT & 5 \\
switch to dasatinib & 2 \\
increase of the imatinib dose & 3 \\
\hline
\end{tabular}

\section{Hematopoietic stem cell transplantation}

Hematopoietic stem cell transplantation (HSCT) was performed in 13 children $(M=9, F=4)$. In 4 patients, the transplant was performed in the $1^{\text {st }}$ CML-CP due to the local preferences of the center. In 3 patients, the reason for HSCT was the advanced phase of CML (CML-BC, $\mathrm{n}=1$; CML-AP, $\mathrm{n}=2$ ), and these children were transplanted in the $2^{\text {nd }} \mathrm{CP}$ (CML-CPII), after TKI treatment. Hematologic toxicity of imatinib was the reason for a transplant in 1 case. In 5 other cases, the loss of molecular and/or cytogenetic response were observed. The donors were either matched unrelated (MUD: $\mathrm{n}=9$ ) or sibling (MSD: $\mathrm{n}=4$ ). Median time from the diagnosis of CML to HSCT was 14.8 months (range 5.7-49 months). In 9 children, HSCT was performed in the $1^{\text {st }} \mathrm{CP}$, and in 3 cases in the $2^{\text {nd }} \mathrm{CP}$. In 1 case, a reduced-intensity conditioning regimen according to CML-SCT I-BFM study was introduced. So far, tests have confirmed $100 \%$ allogenic chimerism and nondetectable BCR-ABL (MR 4.5) in the patients' peripheral blood, but the follow-up period is too short ( 2 months) to confirm the complete success of transplantation.

\section{Follow-up}

Out of 57 patients enrolled in the treatment, 40 patients continue therapy with imatinib, while 17 completed the treatment. Thirty-five patients continue the therapy in pediatric centers (median follow-up: 33 months), and 3 patients in adult centers, with which we are in constant contact (median follow-up: 93 months), while 2 patients were lost to follow-up treatment after being transferred to adult centers (status at the last contact: continuation of the TKI therapy). Among patients who completed the treatment, 13 underwent HSCT (2 patients died due to complications of the post-transplant period), and 4 patients were switched to second-generation TKIs (dasatinib, $\mathrm{n}=3$; nilotinib, $\mathrm{n}=1$ ).

\section{Discussion}

Clinical data concerning the treatment of CML in pediatric population are limited because of its low incidence in children. It should be noted that for the purpose of our analysis we have gathered a relatively large group of patients $(n=57)$ compared with the available literature. The entire observation period amounted for 33 months in patients who continue the treatment in pediatric centers, and 93 months in patients who continue the treatment in adult centers.

\section{Hematopoietic stem cell transplantation}

Before the era of imatinib, HSCT was a standard firstline therapy. Among pediatric patients who underwent transplantation from MSD in the $1^{\text {st }}$ chronic phase (CML-CP) in the years 1982-2004, EFS during the observation period of 3-5 years after HSCT ranged from 61 to $63 \%$. OS ranged from 66 to $87 \% ., 5$ In transplant patients, MUD results were inferior, with EFS ranging from 27 to $55 \%$, and OS from 45 to $65 \% .^{4-7}$ The main cause of death in both groups was acute and chronic GvHD, more common in children after MUD-HSCT. In patients transplanted in the advanced phase (CML-AP or CML-BC) or the $2^{\text {nd }}$ chronic phase (CML-CPII), the results were worse with EFS from 34 to $35 \%$, and OS from 39 to $46 \% .{ }^{6}$ In our study group, only 13 patients underwent HSCT, which was mainly due to the good response to TKI therapy in most cases. OS among these children was $86 \%$. We should remember that despite giving up the use of HSCT as a first-line therapy, it is still the only method by which we can completely eliminate leukemia cells and cure the disease. 


\section{Treatment with imatinib}

One of the first trials with TKI in children was published in 2004 by the Children's Oncology Group and it presented promising results. ${ }^{8}$ For the $2^{\text {nd }}$ phase trial of this study, 31 pediatric patients who experienced failure of interferon therapy were qualified. In this group, all the children achieved CHR, and in $83 \%$ of them CCyR was observed. Another study covered 8 European countries and 30 patients were enrolled in it. ${ }^{9}$ CHR was achieved in $80 \%$ cases, and CCyR in $60 \%$ of the patients enrolled in the trial in the CP and $29 \%$ of the children enrolled in the AP. In half of the children, MMR was reported. Similar results were published in France, in the $4^{\text {th }}$ phase study conducted on a group of 40 patients..$^{10}$ The average duration of the follow-up period in this study was 16 months. CHR within 3 months was achieved in $86 \%$ of the patients, and within 6 months in $98 \%$ of the patients. CCyR was observed in $62 \%$ of the children within 1 year after the inclusion of imatinib therapy, in 34\% of them MMR within 18 months after initiation of the treatment was confirmed. Similar results were achieved in a German clinical trial CML-PAED II published in year 2009. ${ }^{11}$ Out of 42 enrolled patients, 40 achieved CHR within 3 months. In 26 out of 28 patients, CCyR was observed within 1 year after the start of the treatment, and in 17 out of 19, MMR after 18 months from the onset of the treatment. It should be emphasized that all patients from our study group are simultaneously registered in an international database I-CMLPed Study, so far consisting of 351 children diagnosed with CML. This study, aimed at optimizing the treatment of CML in children, is still ongoing. Preliminary results presented at the $56^{\text {th }}$ American Society of Hematology (ASH) Annual Meeting are promising and comparable with the results of treatment achieved in the Polish population. ${ }^{12}$ Our study group consisted of 57 pediatric patients diagnosed in Poland with a median overall follow-up period of 31 months. CHR within 3 months after the implementation of the treatment with imatinib was documented in all patients. CCyR after 12 months of therapy was observed in $69 \%$ of patients, while MMR in $61 \%$ of patients after 18 months of treatment. These results correspond with the quoted literature, in particular with the results of Suttorp et al.'s analysis from 2009, and the results of treatment in the context of clinical trial I-CML-Ped Study. ${ }^{11,12}$ Moreover, when comparing the proportion of patients achieving CCyR and MMR, our results are above promising. However, despite the excellent results of imatinib therapy, one should not forget about HSCT. It is worth noting that HSCT should be considered more frequently in patients diagnosed with CML before puberty due to the fact that long-term TKI intake may in the future be the cause of short stature among these patients. ${ }^{13}$

\section{Side effects of imatinib}

TKI are usually well tolerated; however, some side effects may occur. In most cases, they are classified as mild to moderate and occur mainly in patients in whom TKI therapy was introduced in advanced phases of CML. Nonhematological, relatively common side effects include nausea; vomiting; diarrhea; skin rash; swelling; limb pain; muscle spasms; bone and joint pain; headaches; weight gain; and an increase in liver enzymes. ${ }^{4,9,10,14}$ In one of our patients, we documented severe headaches, which resolved after a temporary decrease in the dose of imatinib. Millot et al. described neutropenia grade 3 or 4 in $27 \%$ of the children receiving imatinib; thrombocytopenia grade 3 or 4 in $5 \%$; and anemia grade 3 or 4 in $2.5 \%$ of the patients. ${ }^{15}$ However, these cytopenias were treatable by temporary discontinuation of the therapy or the administration of granulocyte-colony stimulating factor (G-CSF) in some children with neutropenia. In 5 of our patients, we observed leukopenia, and in 1 thrombocytopenia, which also resolved after a temporary dose reduction of imatinib. Only in 1 case, myelosuppression as a side effect after imatinib implementation was a reason for HSCT. Despite the potential cardiotoxicity, hepatotoxicity, immune disorders and thyroid gland dysfunction observed in adults treated with imatinib, they have not been documented so far in children. ${ }^{16}$ In the group of pediatric patients with CML, the aspect of TKI therapy impact on bone metabolism is very important. Imatinib impairs the differentiation and reduces the activity of osteoblasts and osteoclasts. This can result in growth retardation in children, particularly those who are starting the treatment in prepubertal age. ${ }^{13,17,18}$ In our study group, we observed no impact of imatinib on calcium and phosphate metabolism. We also noticed no abnormalities in serum phosphate, calcium, parathyroid hormone (PTH) and vitamin D levels or tubular function disorders (phosphate absorption). However, we should keep in mind that particular attention should be paid in the group of the youngest patients chronically receiving TKI. They require regular and detailed clinical evaluation, and performing the panel of basic laboratory tests during each visit. Our results confirmed that the recommended daily dose of imatinib is well tolerated in pediatric patients, and severe side effects are relatively rare.

\section{Second-generation TKIs}

$2 \mathrm{G}$ TKIs - dasatinib and nilotinib - were registered for the treatment of adult patients with CML in 2006, and dasatinib only for the therapy of children in 2007. Nilotinib is not currently recommended for the treatment of pediatric patients. $2 \mathrm{G}$ TKIs are recommended when intolerance or resistance to imatinib occur. ${ }^{19,20}$ They are more effective in the treatment of CML due to the linking of both active and inactive conformations of the BCR-ABL protein. In addition, they show greater activity in the case of mutations of $B C R-A B L$ gene, associated with resistance to TKI therapy. ${ }^{21}$ Unfortunately, there are an even more limited number of studies on the use of 2 G TKIs in children. The first reports are from 2011 as the results of the $1^{\text {st }}$ 
phase clinical trial conducted by the Children's Oncology Group. Thirty-nine patients were enrolled for the treatment with dasatinib, including 9 with CML who were resistant to imatinib or who had had an adverse event after using it. In 8 patients, cytogenetic response was observed, in $3 \mathrm{CCyR}$, in $3 \mathrm{PCyR}$, in 1 minor, and in 1 minimal cytogenetic response. In 1 patient, the cytogenetic response was not possible to assess. ${ }^{22}$ The $1^{\text {st }}$ phase study CA 180 018 from 2013 was conducted on a group of 63 pediatric patients, 17 of whom were enrolled in the chronic phase of CML, and only 3 in the advanced phase. Among patients with CML-CP, CHR was achieved in 94\%, CCyR in 82\%, and MMR in $47 \%$. Patients enrolled in the study in the advanced phase of CML achieved slightly worse results. ${ }^{23}$ In our study group, dasatinib was administered in 3 patients, while nilotinib in 1 case. In all 4 cases, these drugs were implemented due to the failure of imatinib treatment. Patients continue the treatment with 2 G TKIs with a satisfactory outcome, and no side effects have been documented. Clinical trials on the use of $2 \mathrm{G}$ TKIs are being carried out and are raising great hopes, especially in patients in whom imatinib has proven to be ineffective.

\section{Discontinuation of the treatment}

Observations in adult patients show that during the TKI therapy and after documenting the undetectable level of BCR-ABL over a period of 24 months or more, one can try to discontinue the treatment with imatinib. ${ }^{24-26}$ In approx. $40 \%$ of these patients, one can confirm continuous MMR despite cessation of the therapy. In our study group, the treatment was discontinued in 1 patient after continuous 26 months of the undetectable level of BCRABL. The patient remained under constant control and, after 11 months of continuous MMR, molecular relapse was confirmed (level of BCR-ABL transcript 3\%). Imatinib was reintroduced and the patient achieved another MMR within 3 months. The results of controlled trials evaluating the efficacy of TKI withdrawal in the groups of pediatric patients are promising, but they have not been published yet. ${ }^{27}$ These results will be very important because of the potential side effects of long-term therapy with imatinib.

\section{Conclusions}

Our results confirm that the introduction of TKI therapy has revolutionized the treatment of CML in the pediatric population by replacing the previous method of treatment with HSCT and allowing a high percentage of OS (96\%) and EFS (81\%). Although the use of TKIs and 2G TKIs is not a cure for CML, and only reduces the risk of disease progression significantly, the results of ongoing clinical trial evaluating the safety of the treatment withdrawal after confirming a continuous 24-month undetectable level of BCR-ABL are promising. Despite the initial enthusiasm due to the excellent results of TKI therapy, there are more reports confirming that the use of imatinib is not devoid of serious side effects. However, in our study group, we observed in only 1 case myelotoxicity WHO grade 4 , which was the reason for HSCT. We should keep in mind the fact that the goal of the therapy in pediatric patients should be rather to cure the disease than to suppress it, which can be achieved only by performing HSCT. In the context of very promising results of HSCT in pediatric patients with CML after reduced-intensity conditioning regimen, HSCT should be taken into consideration, especially in prepubertal children.

\section{References}

1. Millot F, Traore P, Guilhot J, et al. Clinical and biological features at diagnosis in 40 children with chronic myeloid leukemia. Pediatrics. 2005;116(1):140-143.

2. Suttorp M, Millot F. Treatment of pediatric chronic myeloid leukemia in the year 2010: Use of tyrosine kinase inhibitors and stemcell transplantation. Hematology Am Soc Hematol Educ Program. 2010;1:368-376.

3. Andolina JR, Neudorf SM, Corey SJ. How I treat childhood CML. Blood. 2012;119(8):1821-1830.

4. Millot F, Esperou H, Bordigoni P, et al. Allogeneic bone marrow transplantation for chronic myeloid leukemia in childhood: A report from the Société Française de Greffe de Moelle et de Thérapie Cellulaire (SFGM-TC). Bone Marrow Transplant. 2003;32(10):993-999.

5. Cwynarski K, Roberts IA, lacobelli S, et al. Stem cell transplantation for chronic myeloid leukemia in children. Blood. 2003;102(4):1224-1233.

6. Muramatsu H, Kojima S, Yoshimi A, et al. Outcome of 125 children with chronic myelogenous leukemia who received transplants from unrelated donors: The Japan Marrow Donor Program. Biol Blood Marrow Transplant. 2010;16:231-238.

7. Zecca 1, Prete A, Rondelli R, et al. Chronic graft-versus-host disease in children: Incidence, risk factors, and impact on outcome. Blood. 2002;100(4):1192-1200.

8. Champagne MA, Capdeville R, Krailo M, et al. Imatinib mesylate (STI571) for treatment of children with Philadelphia chromosome-positive leukemia: Results from a Children's Oncology Group phase 1 study. Blood. 2004;104(9):2655-2660.

9. Millot F, Guilhot J, Nelken B, et al. Imatinib mesylate is effective in children with chronic myelogenous leukemia in late chronic and advanced phase and in relapse after stem cell transplantation. Leukemia. 2006;20(2):187-192.

10. Millot F, Baruchel A, Guilhot J, et al. Imatinib is efficient but has a negative impact on growth in children with previously untreated chronic myelogenous leukaemia (CML) in early chronic phase (CP): Results of the French national phase IV trial. Blood. 2009;110:863.

11. Suttorp M, Thiede C, Tauer, et al. Chronic myeloid leukemia in pediatrics: First results from study CML-PAED II. Blood. 2009;114:145.

12. Millot F, Guilhot J, Suttorp M, et al. The experience of the International Registry for Chronic Myeloid Leukemia (CML) in Children and Adolescents (I-CML-Ped Study): Prognostic consideration. [Abstract, Oral Presentation No. 521] Session 632: Chronic myeloid leukemia: Prognosis and therapy.

13. Schmid H, Jaeger BA, Lohse J, et al. Longitudinal growth retardation in a prepuberal girl with chronic myeloid leukemia on longterm treatment with imatinib. Haematologica. 2009;94(8):1177-1179.

14. Kolb EA, Pan Q, Ladanyi $M$, et al. Imatinib mesylate in Philadelphia chromosome-positive leukemia in childhood. Cancer. 2003;98:2643-2650.

15. Millot F, Baruchel A, Guilhot J, et al. Imatinib is effective in children with previously untreated chronic myelogenous leukemia in early chronic phase: Results of the French national phase IV trial. J Clin Oncol. 2011;29(20):2827-2832.

16. Kerkela R, Grazette L, Yacobi R, et al. Cardiotoxicity of the cancer therapeutic agent imatinib mesylate. Nat Med. 2006;12(8):908-916. 
17. Millot F, Guilhot J, Baruchel A, et al. Growth deceleration in children treated with imatinib for chronic myeloid leukaemia. Eur J Cancer. 2014;50(18):3206-3211.

18. Jaeger BA, Tauer JT, Ulmer A, et al. Changes in bone metabolic parameters in children with chronic myeloid leukemia on imatinib treatment. Med Sci Monit. 2012;18(12):CR721-728.

19. Kantarjian H, Shah NP, Hochhaus A, et al. Dasatinib versus imatinib in newly diagnosed chronic-phase chronic myeloid leukemia. NEng/ J Med. 2010;362(24):2260-2270.

20. Saglio G, Kim DW, Issaragrisil S, et al. Nilotinib versus imatinib for newly diagnosed chronic myeloid leukemia. $N$ Engl J Med. 2010;362(24):2251-2259.

21. Soverini S, lacobucci I, Baccarani M, et al. Targeted therapy and the T315I mutation in Philadelphia-positive leukemias. Haematologica. 2007;92(4):437-439.

22. Aplenc R, Blaney SM, Strauss LC, et al. Pediatric phase I trial and pharmacokinetic study of dasatinib: A report from the Children's Oncology Group phase I consortium. J Clin Oncol. 2011;29(7):839-844.

23. Zwaan CM, Rizzari C, Mechinaud F, et al. Dasatinib in children and adolescents with relapsed or refractory leukemia: Results of the CA180018 phase I dose-escalation study of the innovative therapies for children with cancer consortium. J Clin Oncol. 2013;31(19):2460-2468.

24. Marangon E, Citterio M, Sala F, et al. Pharmacokinetic profile of imatinib mesylate and $\mathrm{N}$-desmethyl-imatinib (CGP 74588) in children with newly diagnosed $\mathrm{Ph}(+)$ acute leukemias. Cancer Chemother Pharmacol. 2009;63:563-566.

25. Picard S, Titier K, Etienne G, et al. Trough imatinib plasma levels are associated with both cytogenetic and molecular responses to standard-dose imatinib in chronic myeloid leukemia. Blood. 2007;109:3496-3499.

26. Mauro MJ, Deininger MW. Management of drug toxicities in chronic myeloid leukaemia. Best Pract Res Clin Haematol. 2009;22:409-429.

27. Millot $F$, Claviez A, Leverger $G$, et al. Imatinib cessation in children and adolescents with chronic myeloid leukemia in chronic phase. Pediatr Blood Cancer. 2014;61(2):355-357. 